\title{
TABLEAU DES MATIÈRES DANS LES MANUELS DE LANGUES ÉTRANGÈRES ET SON RAPPORT AVEC LE PROGRAMME
}

\begin{abstract}
Résumé
Le sujet de cet article est le sommaire, élément qui dans les manuels de langues étrangères présente des caractéristiques particulières et prend régulièrement la forme de tableau des matières. Selon les principes de la didactique générale, le sommaire doit être en relation directe et linéaire avec les contenus du programme ; celui-ci fournit le cadre référentiel pour la sélection et la répartition des contenus du manuel en indiquant les unités et les segments plus restreints qui correspondent aux leçons dans le manuel. Les unités didactiques sont organisées selon un ordre logique, chronologique ou problématique, qui reflète la structure des savoirs dans le domaine de référence. Dans l'enseignement des langues étrangères, la matière du programme est classifiée selon les types de contenus ou syllabus, alors que dans le tableau des matières des manuels chaque segment intègre des éléments de plusieurs types des contenus. Cela implique le rapport transversal entre le manuel et le programme, dû au fait que les unités didactiques sont conçues par les auteurs du manuel et non par les auteurs du programme. Dans cette recherche, ces constats sont illustrés et justifiés à partir de cinq manuels conformes au programme pour la première année d'enseignement de la deuxième langue étrangère à l'école élémentaire en Serbie. Dans la conclusion, on souligne que les spécificités de la corrélation entre le programme et le manuel devraient être prises en considération dans l'organisation de l'enseignement des langues étrangères en général, et notamment dans l'élaboration, l'analyse et l'utilisation des manuels.
\end{abstract}

Mots-clés : apprentissage des langues étrangères, niveau débutant, programme, manuel, tableau des matières.

*_jovicamikic92@gmail.com 


\section{Introduction}

Dans la tradition pédagogique serbe, le programme est considéré comme le document officiel sur la base duquel on organise l'enseignement des disciplines singulières dans le cadre du système éducatif. Les indications du programme sont concrétisées dans les manuels, matériel didactique principal. Un programme peut donner lieu à plusieurs manuels, ce qui ne met pas en question le rapport entre l'objectif et le moyen : on réalise le programme et le manuel n'est qu'un instrument qui sert à atteindre l'objectif établi. Les enseignants sont obligés à se familiariser avec le programme en vigueur et à s'y tenir dans leur travail ; le manuel est susceptible de modifications, il est changeable et il faut l'utiliser de sorte à réaliser le programme le plus efficacement possible. Or dans l'enseignement des langues étrangères on remarque un phénomène qui implique un écart par rapport à ce modèle général de la corrélation. Au fait :

Lors des entretiens avec les enseignants [des L2] il s'avère souvent qu'ils sont au courant du caractère obligatoire des programmes d'enseignement. Pourtant, les programmes en tant que tels restent inconnus pour eux, vu la conviction dominante qu'il suffit de se tenir aux contenus des manuels, qui sont considérés, à leur tour, comme le matériel créé en conformité avec les exigences du programme. Pour cette raison, de nombreux didacticiens des langues étrangères pensent que pour les enseignants le manuel représente une sorte de "programme d'enseignement cachéc. (Durbaba 2011, 133 ; traduction J.M.)

Il est naturel de se demander dans quelle mesure cette attitude est justifiable et acceptable ; mais il est tout autant pertinent d'examiner les spécificités de la langue étrangère comme matière scolaire, car elles pourraient éclairer les raisons de cette conviction. La question qui représente le point de départ de cette recherche peut donc être formulée de manière suivante : Existe-t-il des caractéristiques du programme et du manuel de langues étrangères, et partant de leur rapport, qui font que les enseignants se reportent principalement aux manuels et négligent dans une certaine mesure le document officiel ?

Dans cette recherche on essaie de trouver des réponses possibles en analysant le tableau des matières, composante qui présente les contenus 
intégrés dans le manuel et permet la comparaison avec les contenus prescrits par le programme. On analyse les manuels de cinq langues étrangères, dans l'intention d'y repérer non seulement le manque de concordance avec les principes de la didactique générale, mais aussi des régularités dans les décalages. On examine le matériel pour la $5^{\text {ème }}$ année de l'école élémentaire, période où les élèves, dans le système éducatif serbe, passent du primaire au collège et obtiennent plusieurs matières nouvelles. Dans la plupart de ces matières, les postulats relatifs à la transposition didactique commencent à se profiler clairement à ce niveau. C'est aussi le niveau qui correspond au début d'enseignement de la seconde langue étrangère et où l'acquisition de la langue étrangère, selon le programme, cède la place à son apprentissage.

\section{Programme, manuel et tableau des matières}

Dans cette partie nous présentons les définitions des concepts principaux dans la littérature pédagogique et didactique serbe, ainsi que leurs spécificités dans l'enseignement des langues étrangères.

On définit le plan d'enseignement comme document qui prescrit les matières, l'ordre de leur introduction et le nombre d'heures attribuées à chaque matière par an et par semaine (Bakovljev 2005, 24 ; Šimleša et Potkonjak 1989, 99). Il est concrétisé dans le programme d'enseignement, qui prévoit les savoirs, les savoir-faire et les attitudes que les élèves doivent acquérir. La structure des contenus du programme doit intégrer "les données scientifiques et théoriques, les conclusions théoriques et les généralisations grâce auxquelles les élèves de l'école élémentaire pourront connaître et comprendre les bases de la science dans son ensemble“ (Makević 2001, 97).

Le manuel est le livre scolaire principal et obligatoire, qui reprend les thèmes et les objectifs mentionnés dans le programme et qui en fait la didactisation supplémentaire (cf. Jakšić 1996, 522 ; Makević 2001, 106 ; Chopin 1992, 16 ; Trebješanin et Lazarević 2001, 16). Les caractéristiques du manuel sont qu'il contient les savoirs systématisées et adaptés au niveau et à l'âge des élèves, afin de contribuer à la construction de leurs savoirs (Ivić, Pešikan et Antić 2008, 27). Le travail des auteurs du manuel est 
facilité grâce au fait que le programme indique les thèmes et les unités didactiques à réaliser, et parfois même le nombre d'heures qu'il faut dédier à chacun des thèmes (Kocić 2001, 137). Dans l'organisation des contenus du manuel, il est nécessaire de respecter les principes suivants : le manuel doit intégrer tous les thèmes et objectifs qui apparaissent dans le programme d'une matière donnée ; il doit se conformer au nombre d'heures prévu ; il doit être représentatif du type des savoirs et des acquis contemporains de la discipline scientifique de référence (Laković 2015, 14).

Le sommaire représente la liste des titres et sous-titres accompagnés de pages correspondantes, une de ces fonctions étant de faciliter l'utilisation du livre. Selon le modèle général du manuel, le sommaire est une sorte de résumé du manuel dans son ensemble : il présente les thèmes dont le manuel traite et indique les notions et les savoirs généraux et spécifiques (Ivić, Pešikan et Antić 2008, 59). Les unités incluses dans le manuel doivent être organisées selon un certain principe - logique, chronologique ou problématique - qui reflète la structure des savoirs dans le domaine de référence et suggère l'importance que les thèmes singuliers ont dans le programme et dans le manuel (idem).

Dans l'enseignement des langues étrangères, tous les trois concepts, ainsi que leurs rapports, présentent des propriétés caractéristiques qui relèvent du statut épistémologique de cette matière scolaire. Au fait, ce qui est appris en classe d'une langue étrangère diffère dans une large mesure des connaissances acquises dans l'apprentissage des matières basées sur la transposition didactique des disciplines scientifiques (cf. Besse 1989, 38 ; Končarević 2002, 34). Une des particularités les plus évidentes du programme concerne la structuration des contenus (cf. Mikić 2014, 13-18) : la matière à enseigner est classifiée selon les types de contenus ou syllabus (grammaire, lexique, fonctions communicatives, etc.), un syllabus pouvant servir de point de départ pour la sélection des autres contenus. À propos de la gradation des contenus, on rencontre des positions divergentes qui ont un impact direct sur le rapport entre le programme et le manuel : certains auteurs retiennent que le programme détermine seulement les catégories de contenus et leurs éléments, d'autres considèrent qu'il prescrit aussi l'ordre de leur introduction (Balboni 1999, 99 ; Cuq 2003, 230 ; Nunan 1988, 158 ; Raičević 2011, 116 ; Richards 2001, 2). 
Les définitions du manuel englobent les livres scolaires ainsi que le matériel utilisé en cours extra-scolaires ou dans l'auto-apprentissage : on entend par manuel tout matériel didactique dans lequel les documents et les activités sont organisés en fonction des objectifs et selon des principes méthodologiques établis (Byram 2004, 626 ; Cuq 2003, 161-162). Puisque dans l'enseignement des langues étrangères on utilise aussi des manuels étrangers, on pourrait considérer comme manuels scolaires même des livres dont l'élaboration n'est pas basée sur le programme.

Dans les manuels récents de langues étrangères on trouve deux types de sommaire. Le premier consiste en une liste de titres des unités didactiques accompagnée des pages correspondantes. Le second type, qui contient des informations relatives aux contenus, prend régulièrement la forme de tableau des matières. Tous les segments didactiques y sont articulés selon des rubriques qui se répètent (thèmes, actes de parole, structures grammaticales, etc.), qui mettent en évidence la manière dont les auteurs ont classifié les contenus. Dans ce type de sommaire, il est possible de suivre parallèlement, au sein de chaque rubrique, la progression des éléments singuliers sélectionnés par les auteurs (cf. Mikić 2014, 54). Le tableau des matières permet par conséquent d'analyser différents aspects de corrélation avec le programme : classification, sélection et gradation des contenus.

\section{Méthodologie de la recherche}

L'objectif de cette recherche est d'éclairer la nature du rapport entre les manuels et le programme des langues étrangères en comparant les tableaux de matières et les contenus prescrits dans les documents officiels. Cet objectif est décliné en recherche des ressemblances et des différences dans les trois aspects évoqués : les syllabus ou types de contenu; les composantes intégrées dans les syllabus particuliers ; la succession des éléments dans chaque type de contenus. À la suite des recherches précédentes (Mikić 2010 ; 2014), nous vérifions l'hypothèse que la corrélation entre le tableau des matières et les contenus du programme est caractérisée par un rapport non-linéaire et qu'il concerne tous les trois aspects.

Le corpus de la recherche est constitué du programme des langues étrangères pour la $5^{\text {ème }}$ année de l'école élémentaire en Serbie (première année d'ap- 
prentissage de la seconde langue étrangère) et des manuels pour les langues allemande, espagnole, française, italienne et russe. Les manuels pour l'espagnol et l'italien sont élaborés en Serbie à partir du programme en vigueur. Parmi les trois autres, publiés à l'étranger, les manuels pour l'allemand et le russe sont adaptés au besoin du publique spécifique, alors que le manuel de français n'a subi aucune modification par rapport à la version originale. Tous les cinq manuels sont accrédités pour l'utilisation dans les écoles en Serbie.

Le recueil des données à propos du programme consiste en un repérage des segments qui font partie de la matière à enseigner ; il s'avère que, en outre du segment Contenus du programme, deux autres parties représentent aussi des syllabus. Le tableau des matières est repéré en principe dans le livre de l'élève, mais dans un cas il figure dans le guide pédagogique. Dans le programme, on sépare les segments valables pour toutes les langues et ceux qui sont propres à chaque langue particulière ; au sein de ce second type de contenus, on cherche des ressemblances et des différences dans les sous-catégories et dans leurs dénominations. Dans les manuels, on répertorie les titres des unités didactiques, les noms des rubriques qui apparaissent dans les unités et les éléments qui figurent dans toutes les rubriques. Les données recueillies sont soumises à l'analyse comparative : pour chacun des cinq manuels, on repère des ressemblances et des différences dans les titres des syllabus ou types de contenus, les éléments choisis pour chacun des syllabus et l'ordre d'introduction des éléments singuliers.

\section{Résultats de la recherche}

Nous présentons d'abord l'organisation des contenus dans le programme et ensuite les tableaux des matières dans les manuels particuliers. Pour rendre la comparaison plus transparente, nous traduisons en français tous les noms des syllabus et des rubriques, en gardant la langue originale seulement pour les titres des unités didactiques dans les manuels.

\section{Les contenus dans le programme pour les langues étrangères}

Le programme englobe trois types de contenus ou syllabus. Les noms et les éléments des deux premiers (thèmes et situations et fonctions 
communicatives) sont fournis en langue serbe et ils sont identiques pour toutes les langues étrangères. La troisième partie, intitulée Contenus $d u$ programme, comporte le sous-titre Contenus morphosyntaxiques et phonétiques avec exemples; on y constate des écarts entre les langues dans la catégorisation, sélection et classification des contenus (les catégories grammaticales ne sont pas les mêmes, elles ne sont pas déclinées de la même manière, les éléments de phonétique et d'orthographe n'existent pas dans toutes les langues, la terminologie pour la langue russe dénote un type spécifique de grammaire de référence). Toutes les catégories grammaticales sont données en langue serbe et les exemples sont fournis, évidemment, en langues étrangères.

\section{Les tableaux des contenus dans les manuels}

\section{Manuel d'allemand : Wir 1}

Le tableau des contenus est composé de quatre modules, qui contiennent trois ou quatre leçons et des segments dédiés aux activités langagières, à la grammaire et à l'autoévaluation.

La catégorisation des contenus dans le tableau des matières comporte un seul élément identique au programme (Grammaire) ; les noms des autres rubriques (Communication, Textes, jeux, chanson) ne figurent pas dans le programme comme titres des syllabus. Les titres des modules et des leçons peuvent être interprétés comme l'exemplification de certains thèmes, situations ou fonctions communicatives du programme : $\mathrm{Ma} \mathrm{fa}$ mille - Das ist meine Familie ; lieu et rue où j'habite - Wo wohnt Ihr?: animaux domestiques - Mautzi, unsere Katze.

Les phénomènes les plus importants qu'on constate à propos de la sélection sont les suivants :

-apparition des éléments de deux ou trois syllabus du programme dans chacune des unités du manuel ;

- correspondance partielle ou séparation des éléments grammaticaux du programme : Utilisation productive du nominatif, datif et accusatif au singulier et du nominatif et accusatif au pluriel, avec ou sans prépositions - accusatif : article, accusatif de l'article indéfini, accusatif de l'article défini, pronoms personnels : accusatif au singulier et au pluriel; 
-récurrence des éléments grammaticaux dans plusieurs modules : présent ; nominatif et accusatif; article possessif;

- apparition de certains thèmes ou fonctions communicatives dans la rubrique Textes, jeux, chansons : on décrit l'appartement; on salue; on répond à l'invitation;

-inclusion d'éléments qui ne figurent pas dans le programme : on lit une fable illustrée; on fait les mots croisés; on imagine un dialogue.

L'ordre d'introduction des éléments dans le manuel est différent par rapport aux éléments de tous les trois syllabus du programme.

\section{Manuel d'espagnol : ¿Hablamos? 1}

Le tableau des contenus est composé de huit unités didactiques.

Dans la catégorisation des contenus on constate des points identiques (Fonctions communicatives, Contenus grammaticaux) et des ajouts par rapport au programme (Contenus culturels). Les titres des unités peuvent être interprétés comme l'exemplification de certains thèmes, situations ou fonctions communicatives du programme : fêtes - iFeliz cumpleaños! ; moi et mes camarades - Dime con quien vas y te diré quien éres; lieu et rue où j'habite-Caminando por la calle....

Les phénomènes les plus importants qu'on constate à propos de la sélection sont les suivants :

-apparition des éléments de tous les trois syllabus du programme dans chacune des unités du manuel ;

-intégration des éléments des syllabus Thèmes et situations et Fonctions communicatives dans la rubrique Fonctions communicatives : dire les jours de la semaine; parler de la nourriture; nommer les parties de la maison; se présenter et présenter les autres;

- correspondance des éléments du syllabus grammatical : présent des verbes ser et estar; déterminants possessifs; adverbes de lieu;

- omission de certains éléments du syllabus grammatical : pluriel des adjectifs ; pronom réfléchi ; pretérito perfecto simple ;

- apparition d'éléments qui ne figurent pas dans le programme : envoyer des messages par le portable; chatter sur Internet ; parler du climat ;

-apparition de certains éléments grammaticaux, avec des exemples 
différents, dans plusieurs unités du manuel : présent ; nombres ; exclamations ;

-spécification du thème les données principales sur le/ les pays dont on apprend la langue: Barcelone, carnavals, costumes typiques.

L'ordre d'introduction des éléments dans le manuel est différent par rapport aux éléments de tous les trois syllabus du programme.

\section{Manuel de français : Pixel 1}

Le tableau des matières est composé d'un segment introductif et de six unités didactiques, dont chacune comporte trois leçons et les parties dédiées aux contenus culturels et à l'évaluation.

La catégorisation des contenus dans le tableau comporte des ressemblances et des différences par rapport aux contenus du programme : le syllabus grammatical est couvert par deux rubriques dans le manuel (Grammaire et Conjugaison) ; le syllabus Thèmes et situation est représenté par la rubrique Thème-lexique; le syllabus Fonctions communicatives peut être mis en relation avec la rubrique Objectifs de communication; les titres des deux autres rubriques (Phonétique, Civilisation) n'apparaissent pas dans le programme comme syllabus séparés. Les titres des unités et des leçons peuvent être interprétés comme l'exemplification des thèmes, situations ou fonctions communicatives : école - La rentrée, le collège ; exprimer le temps - Quelle heure est-il ?, Quel temps fait-il ?; fêtes - Joyeux anniversaire!.

En ce qui concerne la sélection des éléments, on constate les phénomènes suivants :

- apparition des éléments de tous les trois syllabus du programme dans chacune des unités du manuel ;

-correspondance de certains éléments : les mois de l'année; se présenter et présenter les autres; futur proche;

- omission de certains éléments du programme : les pièces de la maison ; exprimer une interdiction et réagir à une interdiction ; passé composé ;

-inclusion d'éléments qui ne figurent pas dans le programme : les transports; donner son emploi du temps ;

-spécification du thème les données principales sur le/ les pays dont on apprend la langue : Francophonie : où est-ce qu'on parle français ?; 
Le collège en France ; J'adore ma ville! (Montréal, Bruxelles, Dakar).

L'ordre d'introduction des éléments dans le manuel est différent par rapport aux éléments de tous les trois syllabus du programme.

\section{Manuel d'italien : Amici 1}

Le tableau des contenus est composé de huit unités didactiques.

La catégorisation des contenus dans le tableau présente des points communs et des écarts par rapport aux programme : le syllabus Thèmes et situations est représenté dans le manuel par la rubrique Thème-lexique; le syllabus Fonctions communicatives correspond à la rubrique homonyme dans le manuel ; l'homologie existe entre le syllabus grammatical et la rubrique Structures langagières ; les titres des deux autres rubriques (Civilisation et culture, Orthographe et phonétique) n'apparaissent pas dans le programme comme syllabus à part. Les titres des unités peuvent être interprétés comme l'exemplification de certains thèmes, situations ou fonctions communicatives du programme : école - Una nuova alunna; fêtes - Buon Natale a tutti!; ma maison-Casa dolce casa.

À propos de la sélection des éléments on constate les phénomènes suivants :

-apparition des éléments de tous les trois syllabus du programme dans chacune des unités didactiques du manuel ;

- correspondance presque totale dans les trois syllabus : vêtements ; exprimer la possession et l'appartenance; impératif;

- omission de certains éléments du syllabus grammatical : position de l'article et de la préposition avec l'adjectif indéfini tutto ; proposition temporelle;

-répétition de certaines fonctions communicatives dans plusieurs unités : identifier et nommer des personnes; attirer l'attention; exprimer des sensations physiques et des besoins;

-spécification du thème les données principales sur le/ les pays dont on apprend la langue : caractéristiques géographiques de l'Italie; importance de la mode en Italie; physicien italien Guglielmo Marconi.

L'ordre d'introduction des éléments dans le manuel est différent par rapport aux éléments de tous les trois syllabus du programme. 


\section{Manuel de russe : Конечно! 1}

Le tableau des contenus est composé de sept unités didactiques articulées en trois ou quatre leçons.

Dans la catégorisation des contenus on constate, avec quelques différences terminologiques, l'adéquation entre le programme et le manuel : Thèmes et situations - Thèmes ; Fonctions communicatives Compétences communicatives ; Grammaire - Outils grammaticaux et langagiers. Les titres des unités et des leçons peuvent être interprétés comme l'exemplification de certains thèmes, situations ou fonctions communicatives du programme : se présenter - Меня зовуй... ; е́cole - Шко́ла No 3 ; fêtes - Любимьге ирразяники.

Les phénomènes les plus importants qu'on constate à propos de la sélection sont les suivants :

-apparition des éléments de deux ou trois syllabus du programme dans chacune des unités didactiques du manuel ;

-intégration des thèmes, situations et fonctions communicatives dans la rubrique Compétences communicatives : se présenter; comment décrire les vêtements; parler d'une ville;

- apparition des éléments du syllabus Thèmes et situations dans la rubrique Outils grammaticaux et langagiers : vêtements ; quelques fêtes ; les noms des mois et des saisons;

- différences dans la dénomination et la présentation des éléments grammaticaux : exprimer la possession-pronoms possessifs à la $3^{\text {ième }}$ personne du pluriel ; exprimer des relations spatio-temporelles - prépositions в et $\mathrm{на}$;

-inclusion d'éléments grammaticaux qui ne figurent pas dans le programme : infinitif; le présent des verbes pronominaux.

L'ordre d'introduction des éléments dans le manuel est différent par rapport aux éléments de tous les trois syllabus du programme.

\section{Conclusion}

L'analyse effectuée confirme la supposition relative à la nature spécifique du rapport entre le programme et le manuel dans l'enseignement des langues étrangères. Les écarts par rapport au modèle général sont évidents déjà dans le programme en tant que tel : il existe plusieurs syllabus 
complémentaires mais hétérogènes et la classification des contenus n'est reliée au domaine scientifique de référence que dans la grammaire.

À propos de la catégorisation des contenus dans les manuels on constate les régularités suivantes : les titres des unités ont un rapport métonymique avec des thèmes, situations ou fonctions communicatives du programme ; la dénomination des types de contenus comporte des points communs avec le programme, mais la coïncidence totale n'existe dans aucun des manuels analysés. La sélection des éléments des syllabus présente aussi plusieurs particularités : une unité didactique du manuel englobe les éléments de tous les syllabus du programme ; tous les éléments des syllabus du programme n'apparaissent pas dans les tableau et parfois on y inclut des éléments qui ne figurent pas dans le programme ; certains éléments apparaissent dans plusieurs unités didactiques ; les données sur le pays dont on apprend la langue figurent généralement dans toutes les unités didactiques. En ce qui concerne l'ordre d'introduction des éléments dans le manuel, on constate des différences entre les manuels particuliers et entre tous les manuels et le programme.

Pour toutes ces raisons, on peut affirmer que les syllabus et leurs éléments dans le programme ne représentent pas des unités dans le sens qu'on attribue à cette notion dans la didactique générale. L'unité didactique, entendue comme un segment de contenus autour duquel on élabore un ensemble d'activités cohérent (cf. Cuq 2003, 242), n'est pas créée par les auteurs du programme, mais par les auteurs de manuels.

Ce constat peut expliquer le fait que les enseignants des langues étrangères se réfèrent au manuel plutôt qu'au programme. Le document officiel offre des éléments à enseigner, mais les enseignants ne sont pas en position, comme leurs collègues enseignants d'autres matières, de les réaliser séparément et selon la succession indiquée. Même si on prend comme point de départ les objectifs du programme, reste le fait que leur réalisation nécessite de travailler sur certains contenus, d'en faire la sélection, la gradation, l'exemplification et la didactisation. Préparer le travail en classe en se tenant au programme oblige l'enseignant à effectuer certaines tâches qui incombent habituellement aux auteurs des manuels.

Une autre implication didactique concerne la coordination du travail des enseignants des langues étrangères et des enseignants d'autres matières scolaires. Pour éviter une juxtaposition mécanique des objectifs, 
contenus et activités, il semble indispensable que les enseignants prennent connaissance de la nature des programmes et des manuels des disciplines respectives ; cette nécessité concerne toute forme d'enseignement institutionnel, et elle est notamment accentuée dans l'enseignement bilingue. Les spécificités du rapport entre le manuel et le programme concernent particulièrement les activités liées à la conception, élaboration, analyse, accréditation et utilisation des manuels de langues étrangères ; toutes ces opérations devraient être effectuées selon des critères pertinents, qui s'écartent partiellement des modèles de la didactique générale.

Nous signalons à la fin que notre recherche ne peut pas donner lieu à des généralisations car elle est inévitablement contextualisée. La corrélation entre le manuel et le programme de langues étrangères dépend principalement de la conception du programme dans un environnement particulier et dans un espace de temps donné. Au moment de la rédaction de cet article, le programme des langues étrangères à l'école élémentaire en Serbie est en train de changer sa structure, ce qui implique le caractère dynamique de la corrélation. En tous cas, il sera toujours le plus pertinent de l'analyser à partir des tableaux des matières dans les manuels.

\section{Références bibliographiques}

\section{Corpus}

Amštajn-Baman, Kristine, Ulf Borgvart, Monika Broš, Elena DenisovaŠmit, Danuta Genč, Peter Jakubov, Gizela Rajhert-Borovski, Evelin Valah, Dragana Kerkez et Jelena Ginić. 2015. Konečno! 1: Ruski jezik za peti razred osnovne škole: Udžbenik za prvu godinu učenja. Beograd: Klett.

Blatešić, Aleksandra. 2007. Amici 1: Italijanski jezik za 5. razred osnovne škole: Prva godina učenja: Priručnik za nastavnike. Beograd : Zavod za udžbenike.

Favret, Catherine. 2011. Pixel 1: Méthode de français: Livre de l'élève. Paris : CLE International/ Beograd : Data Status.

Mota, Đorđo. 2006. Wir 1: Nemački jezik za 5. razred osnovne škole: Prva godina učenja. Beograd: Klett. 
Pejović, Anđelka, et Maja Andrijević. 2007. ¿Hablamos? 1: Španski jezik za peti razred osnovne škole: Prva godina učenja. Beograd: Zavod za udžbenike.

Pravilnik o nastavnom planu za drugi ciklus osnovnog obrazovanja i vaspitanja i nastavnom programu za peti razred osnovnog obrazovanja i vaspitanja. Službeni glasnik RS - Prosvetni glasnik, br. 6/07.

\section{Bibliographie}

Bakovljev, Milan. 2005. Didaktika. Sombor: Učiteljski fakultet.

Balboni, Paolo. 1999. Dizionario di glottodidattica. Perugia: Guerra/ Welland: Soleil.

Besse, Henri. 1989. "De la relative rationalité des discours sur l'enseignement / apprentissage des langues." Langue française 82: 28-43.

Byram, Michael, ed. 2004. Routledge Encyclopedia of Language Teaching and Learning. London/New York: Routledge.

Chopin, Alain. 1992. Les manuels scolaires : histoire et actualité. Paris : Hachette.

Cuq, Jean-Pierre. 2003. Dictionnaire de didactique du français langue étrangère et seconde. Paris : CLE International/SEJER.

Durbaba, Olivera. 2011. Teorija i praksa učenja i nastave stranih jezika. Beograd: Zavod za udžbenike.

Ivić, Ivan, Ana Pešikan et Slobodanka Antić. 2008. Vodič za dobar udžbenik: Opšti standardi kvaliteta udžbenika. Novi Sad: Platoneum.

Jakšić, Anka, ed. 1996. Pedagoški leksikon. Beograd: Zavod za udžbenike i nastavna sredstva.

Kocić, Ljubomir. "Didaktičko-metodički zahtevi u oblikovanju strukture udžbenika.” In Savremeni osnovnoškolski udžbenik: Teorijsko-metodološke osnove, edité par Biljana Trebješanin et Dušanka Lazarević, 131-156. Beograd: Zavod za udžbenike i nastavna sredstva.

Končarević, Ksenija. 2002. Savremeni udžbenik stranog - ruskog jezika: Struktura i sadržaj. Beograd: Zavod za udžbenike i nastavna sredstva. Laković, Zoran. 2015. Put do dobrog udžbenika: Metodološki okvir za izradu, utvrđivanje i praćenje kvaliteta udžbenika. Podgorica: Zavod za udžbenike i nastavna sredstva.

Makević, Sreten. 2001. Pedagoško-didaktički principi i kriterijumi u izra- 
di nastavnih planova i programa. Šabac: Viša škola za obrazovanje vaspitača.

Mikić, Jovica. 2010. "Specifičnost udžbenika za strane jezike. Analiza strukturno-funkcionalnih komponenti." Inovacije u nastavi 23 (1): 87-96.

Mikić, Jovica. 2014. Korelacija udžbenika i programa u nastavi stranih jezika u osnovnim školama Srbije. Thèse de doctorat, Université de Belgrade, Faculté de Philologie.

Nunan, David. 1988. Syllabus Design. Oxford: Oxford University Press.

Raičević, Vučina. 2011. Rečnik lingvodidaktičke terminologije. Beograd: Zavod za udžbenike.

Richards, Jack. 2001. Curriculum Development in Language Teaching. Cambridge: Cambridge University Press.

Šimleša, Petar et Nikola Potkonjak, ed. 1989. Pedagoška enciklopedija (Vol 2). Beograd : Zavod za udžbenike i nastavna sredstva.

Trebješanin, Biljana, et Dušanka Lazarević. 2001. Savremeni osnovnoškolski udžbenik: Teorijsko-metodološke osnove. Beograd: Zavod za udžbenike i nastavna sredstva.

\section{Jovica Mikić}

\section{PREGLED SADRŽAJA U UDŽBENICIMA STRANIH JEZIKA I NJEGOV ODNOS SA PROGRAMOM}

\section{Sažetak}

Tema ovog rada jeste pregled sadržaja, komponenta koja u udžbenicima stranih jezika ima posebne odlike i uobičajeno se predstavlja u vidu tabele. Prema pedagoškodidaktičkim načelima, pregled sadržaja treba da bude povezan sa programom na direktan i linearan način; program pruža referentni okvir za izbor i raspoređivanje sadržaja u udžbeniku, tako što navodi nastavne celine i uže odeljke koji odgovaraju udžbeničkim lekcijama. Nastavne celine organizovane su prema logičkom, hronološkom ili problemskom principu, koji odražava strukturu znanja u odgovarajućoj naučnoj oblasti. U nastavi stranih jezika, građa u programu je razvrstana prema tipovima sadržaja ili silabusima; što 
se tiče pregleda sadržaja u udžbeniku, u svaki nastavni segment uključeni su elementi iz više tipova sadržaja. To znači da između programskih i udžbeničkih sadržaja postoji transverzalan odnos, a razlog je činjenica da nastavne celine osmišljavaju pisci udžbenika a ne autori programa. Ova postavka u radu se ilustruje i dodatno obrazlaže na osnovu korpusa sačinjenog od pet udžbenika izrađenih u skladu sa programom za prvu godinu nastave drugog stranog jezika u osnovnim školama u Srbiji. U zaključku se ističe da posebne odlike korelacije između programa i udžbenika treba imati u vidu u organizaciji nastave stranih jezika uopšte, a posebno pri izradi, analizi i korišćenju udžbenika.

Ključne reči: učenje stranih jezika, početni nivo, program, udžbenik, pregled sadržaja. 